\title{
Review of Multi-Scale Electromagnetic Modeling
}

\author{
W. C. Chew ${ }^{1,2}$, L.J. Jiang ${ }^{1}$, H.Y. Chao ${ }^{3}$, A. J. Hesford ${ }^{4}$, M.K. Li ${ }^{5}$, Z.G. Qian ${ }^{6}$, Y.G. Liu ${ }^{1}$, Y.P

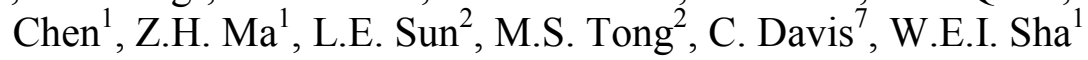

\begin{abstract}
This paper reviews various methods to solve multiscale problems ranging from low-frequency methods to very high-frequency methods.
\end{abstract}

\section{INTRODUCTION}

Solving electromagnetics problems is a challenging task, especially when the structure is multi-scale. A challenging problem in computational electromagnetics is in solving problems in the lowfrequency regime, especially the regime between static and electrodynamics. When the wavelength is much longer than the size of the structure, circuit physics prevails. When the wavelength is sizeable compared to the structure, wave physics becomes important, and a simulation method has to capture the wave physics interaction. When a structure is multi-scale, then both circuit physics and wave physics are important. A simulation method has to capture both physics.

In this presentation, we will discuss various methods to solve the multi-scale problems, starting with the use of preconditioners, followed by the use of the equivalence principle algorithm (EPA). Then we discuss the use of the augmented electric-field integral equation (A-EFIE) method for various problems. Finally, we discuss solution methods in the shortwavelength limit.

\section{PRECONDITIONER AND PARALLEL COMPUTING}

One can also solve multi-scale problems by the use of a preconditioner such as the self-box inclusion method $[1,2]$. When a geometry has multiple scales and is discretized into elements of disparate sizes, the pertinent matrix has a large range of eigenvalues, giving rise to an ill-conditioned matrix system. The small length scales usually give rise to large eigenvalues that can eclipse other eigenvalues significant to the problem. One way to remove the large eigenvalues is by designing a self-box inclusion preconditioner. In this method, an inclusion box is designed around geometry with fine meshes. The inversion of the matrix relevant to the fine meshes is then used as a preconditioner. We have found that this can significantly reduce the condition number of the matrix system, and allow convergence by iterative solvers.

A parallel computer code [2] has been developed making use of these concepts. As a result, multi-scale problems with several million unknowns have been solved (see Figure 1).
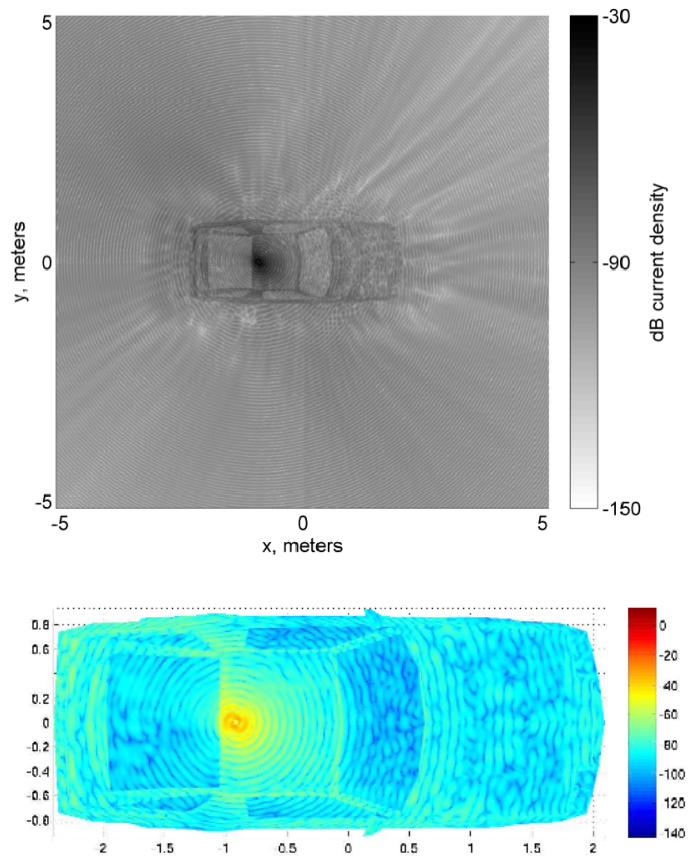

Figure 1. A multi-scale problem involving an XM antenna mounted on a car which is in turn mounted on a ground plane. Parallel computing with MLFMA and 3.6M unknowns were used.

\section{EQUIVALENCE PRINCIPLE ALGORITHM}

The equivalence principle algorithm (EPA) $[3,4]$ is a good way to decompose a larger problem into smaller problem domains. It also allows regions of circuit physics to be separated from the regions of wave physics. The use of EPA allows a large problem to be decomposed into a sum of smaller problems, so that only smaller problems need to be solved at one time. Then the solution to the larger problem is accomplished by rigorously stitching the smaller problems together.

Recently, we have developed an EPA that allows the equivalence surface to cut through metal, and break an object involving metal into smaller objects (Figure 2).

Figure 3 shows the use of EPA to simulate the multiscale problem of an XM antenna on top of a car. The unknown count involved for EPA is 355,305 , and the code has been accelerated with an eight-level MLFMA. GMRES with 50 restart was used to reduce the residual error to $2.0 \mathrm{E}-2$ after 200 iterations. The 
total memory usage was $2.6 \mathrm{~GB}$, and the computer used was a single processor Dell Precision 670, taking $54.2 \mathrm{~s}$ per iteration.

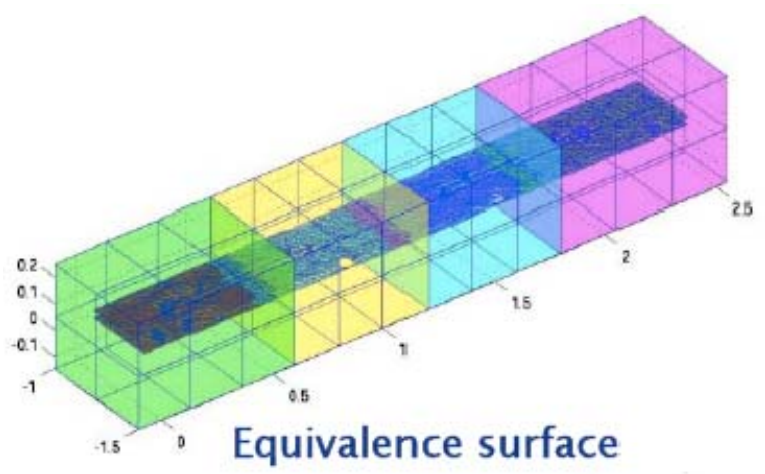

Figure 2. Equivalence surfaces are used to break a large object into smaller objects to facilitate easier solutions.

We can also use the EPA concept to break a large 30 by 30 antenna array into tiny problems (Figure 4). The antenna array problem constitutes 7.2 million unknowns. Using EPA, the unknown count is reduced to 0.86 million (since only unknowns on an equivalence surface are needed) with total memory usage of $12 \mathrm{~GB}$. The problem can be solved to $2.0 \mathrm{E}-2$ precision with 149 iterations on a Dell Precision 690 with Intel XEON $3 \mathrm{GHz}$ computer.
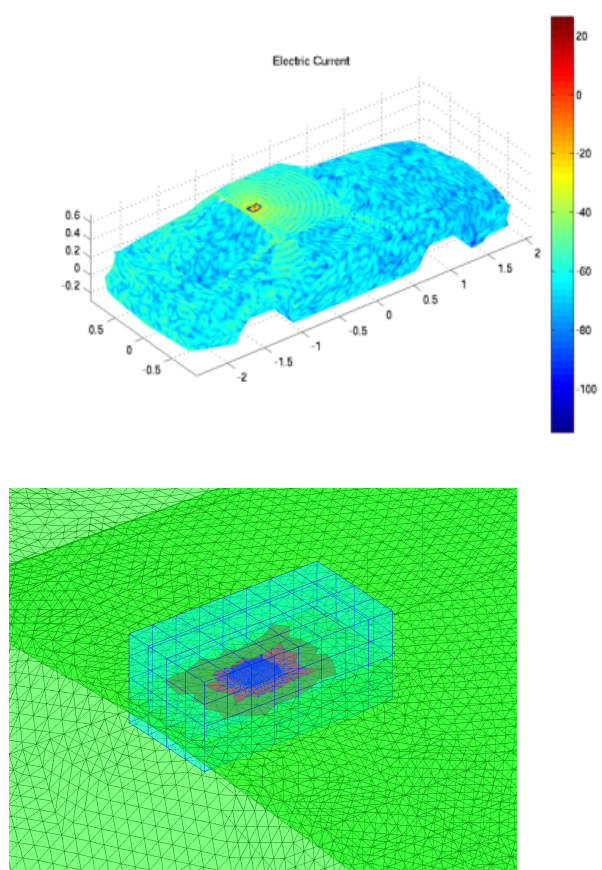

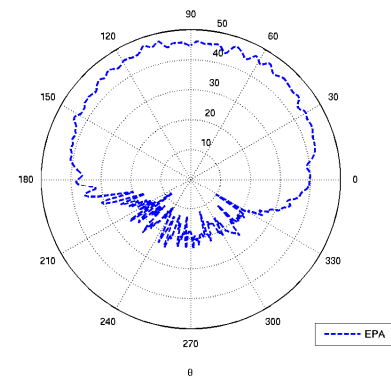

Figure 3. Multi-scale simulation of an XM antenna on a car, where the radiation pattern can be calculated.

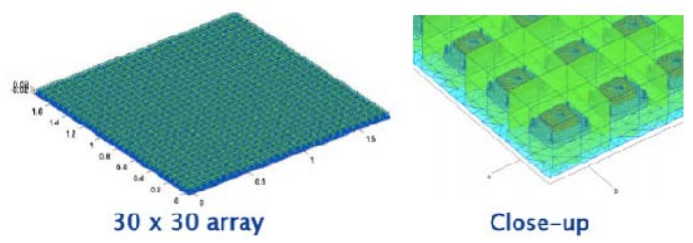

Figure 4. A large antenna array can be broken into tiny pieces using EPA allowing a large problem to be solved.

\section{AUGMENTED ELECTRIC FIELD INTEGRAL EQUATION}

Recently, we have developed an augmented electric field integral equation (A-EFIE) for low-frequency electromagnetics problems $[5,6]$. It reformulates the mixed potential integral equation to be in a generalized saddle-point form by explicitly enforcing the current continuity equation and including the charge as additional unknowns $[7,8]$. We solved the final dense matrix equation using iterative methods, accelerated the matrix-vector product with mixed-form fast multipole algorithm [9], and developed an efficient constraint preconditioner for fast convergence. This technique avoids the complexity of the conventional loop-tree decomposition method and solves complicated electromagnetic problems with greatly enhanced performance.

In Figure 5, we show the simulation of a spiral inductor with four different levels of mesh density. Low frequency breakdown is usually accompanied by non-convergence of iterative methods. The four AEFIE simulations show consistent convergence as the mesh size decreases and the number of unknowns increases. They all give the same inductance value at $0.62 \mathrm{nH}$, which agrees with the loop-tree decomposition method.

The simulation of a full package shown in Figure 6 demonstrates the great performance of the A-EFIE method. It is a complicated real-world problem with over one million unknowns. It can be solved in about 1.5 hours on a $3 \mathrm{GHz}$ single CPU desktop computer using 6.2 GB memory. 


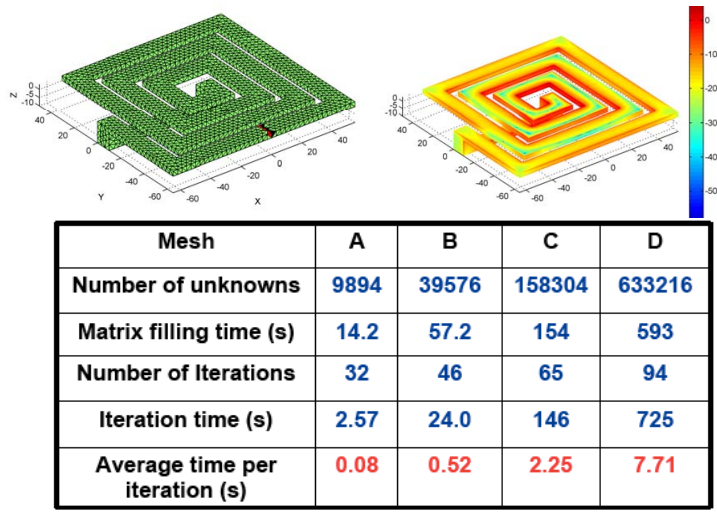

Figure 5. Simulation of a spiral inductor with increasing mesh density with no sign of low-frequency breakdown.
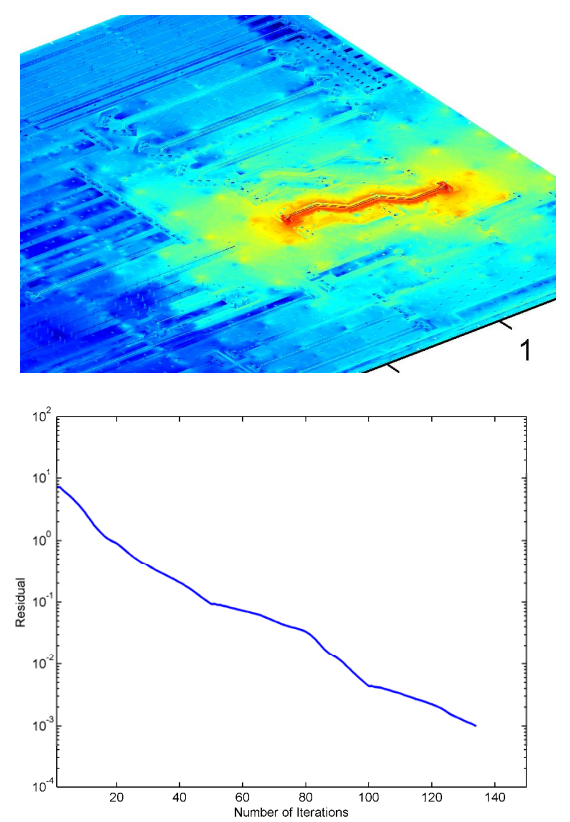

Figure 6. A full package simulation. There are four layers and 222 nets. Surface electric current distribution on the full package is in $\mathrm{dB}$ scale. Unit: $\mathrm{A} / \mathrm{m}$. The discretization has 1007 691 inner edges. Geometry unit: (top) The close-up of the current is around the excitation. (bottom) The iteration history is shown. GMRES reduces the residual error to $1 . \mathrm{E}-3$ with 134 iterations.

Recently, we have improved the A-EFIE using the vector addition theorem. This can result in $25 \%$ savings in memory when solving low-frequency problems, and at the same time, prevent the onset of low-frequency breakdown [10] (see Table 0 ). The AEFIE concept has also been extended to the layered medium Green's function approach [11].

\begin{tabular}{|l|l|l|l|l|l|} 
Method Number of & Memory of & Memory of Rad. & Number of & Average Time \\
\hline
\end{tabular} \begin{tabular}{|c|c|c|c|c|c|}
\hline LF-FMA & 7 & $0.616 \mathrm{MB}$ & $515.27 \mathrm{MB}$ & 134 & $17.87 \mathrm{~s}$ \\
\hline
\end{tabular}

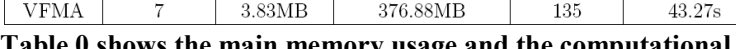
cost of the VFMA with vector addition theorem and the LFFMA for solving A-EFIE in the full package simulation.

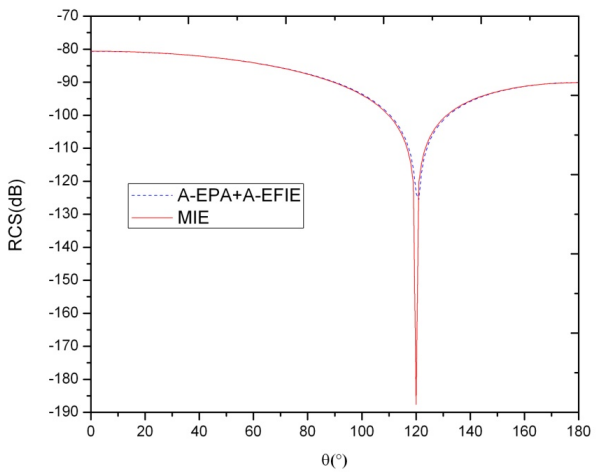

Figure 7. The use of A-EPA with A-EFIE for a low-frequency scattering problem.

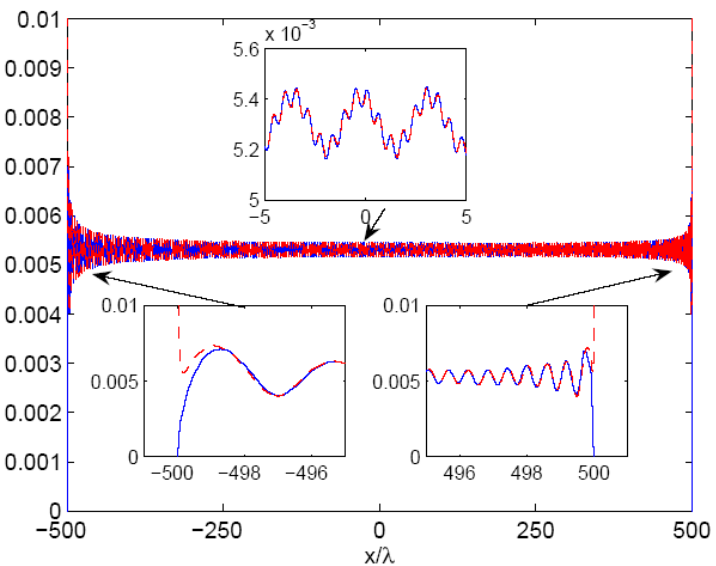

\begin{tabular}{|c|c|c|c|}
\hline Width & Matrix Fill & Post-Processing & Scattering Error \\
\hline $0.5 \lambda$ & $13.6 \mathrm{~s}$ & $0.25 \mathrm{~s}$ & $0.02 \%$ \\
\hline $1.0 \lambda$ & $15.1 \mathrm{~s}$ & $0.27 \mathrm{~s}$ & $0.08 \%$ \\
\hline $2.0 \lambda$ & $15.1 \mathrm{~s}$ & $0.34 \mathrm{~s}$ & $0.12 \%$ \\
\hline $4.0 \lambda$ & $20.1 \mathrm{~s}$ & $0.44 \mathrm{~s}$ & $0.14 \%$ \\
\hline $7.9 \lambda$ & $20.3 \mathrm{~s}$ & $0.62 \mathrm{~s}$ & $0.27 \%$ \\
\hline $15.8 \lambda$ & $22.1 \mathrm{~s}$ & $0.99 \mathrm{~s}$ & $0.18 \%$ \\
\hline $31.6 \lambda$ & $22.5 \mathrm{~s}$ & $1.73 \mathrm{~s}$ & $0.19 \%$ \\
\hline $63.0 \lambda$ & $22.8 \mathrm{~s}$ & $3.19 \mathrm{~s}$ & $0.20 \%$ \\
\hline $126.0 \lambda$ & $23.8 \mathrm{~s}$ & $6.11 \mathrm{~s}$ & $0.20 \%$ \\
\hline $251.0 \lambda$ & $23.9 \mathrm{~s}$ & $12.0 \mathrm{~s}$ & $0.22 \%$ \\
\hline $501.0 \lambda$ & $23.7 \mathrm{~s}$ & $23.5 \mathrm{~s}$ & $0.24 \%$ \\
\hline $1000.0 \lambda$ & $24.1 \mathrm{~s}$ & $46.8 \mathrm{~s}$ & $0.24 \%$ \\
\hline
\end{tabular}

TABLE I

RUN-TIMES (IN SECONDS) AND SCATTERING ERRORS FOR THE NON-UNIFORM, $N=20$ MOM DATA POINTS OF FIG. 7. THE INCREASE IN POST-PROCESSING TIME IS UNAVOIDABLE, SINCE WE ARE CONSIDERING A NUMBER OF SCATTERING ANGLES PROPORTIONAL TO $k$. BECAUSE THE CURRENT SOLUTION IS OBTAINED WITH A FIXED COST AND THE ERROR IS BOUNDED, WE CLAIM THAT THE ALGORITHM IS FREQUENCY INDEPENDENT

Figure 8. Total current for a 1000 wavelength strip using $\mathbf{N}=\mathbf{3 0}$ nodes, and a minimally-varying MOM. The numerical solution agrees with GTD away from the strip edges, where GTD breaks down. The table shows the frequency independence of the runtimes.

We have also developed an augmented EPA (A-EPA) with A-EFIE to allow the solution of really low frequency problems (see Figure 7). 


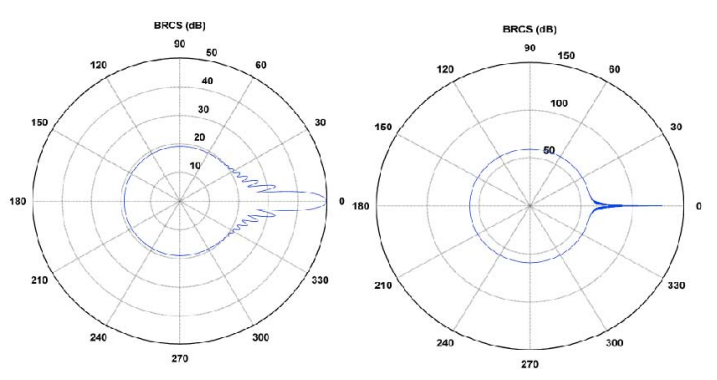

Figure 9. Frequency independent calculation of a sphere scattering solution (left) a 5 wavelength sphere, (right) a 500 wavelength sphere.

\section{SHORT-WAVELENGTH PROBLEMS}

The other extreme of multi-scale problems is the shortwavelength problems where the size of the scatterer is very much larger than the wavelength. This problem has been addressed by many researchers in the mathematics community $[12,13,14]$. In a numerical method based on the method of moments [16,17], the computer time increases with frequency. Even though very large problems have been solved by numerical methods [18,19], they involve the use of supercomputers. However, the physics of these problems necessitate that the solution method should be frequency independent for short wavelengths.

Recently, we have shown that the scattering solution of a strip using a frequency independent technique [20]. The scattering solution of a sphere can also be calculated in a frequency independent manner [21].

\section{CONCLUSIONS}

Computational electromagnetics serves to produce new simulation tools for next-generation engineering design and prototyping. Its new direction lies in its ability to perform multi-physics and multi-scale calculations. Computational electromagnetics will augment traditional pencil and paper calculations.

\section{References}

1. H.Y Chao, "A multilevel fast multipole algorithm for analyzing radiation and scattering from wire antennas in a complex environment," Ph.D. Thesis, Dept. ECE, U. Illinois, Urbana-Champaign, January 2003.

2. A.J. Hesford and W. C. Chew, "On Preconditioning and the Eigensystems of Electromagnetic Radiation Problems", IEEE Transactions Antennas Propag., vol. 56, pp. 2413--2420, August 2008.

3. M. K. Li and W. C. Chew, "A Domain Decomposition Scheme Based on Equivalence Theorem," Micro. Opt. Tech. Lett., v. 48, no. 9, pp. 1853-1857, Sept. 2006.

4. M. K. Li and W. C. Chew, "Wave-Field Interaction with Complex Structures Using Equivalence Principle Algorithm," IEEE Trans. Antenn. Propag., vol. 55, no. 1, pp. 130-138, 2007

5. Z. G. Qian and W. C. Chew, "An augmented EFIE for high-speed interconnect analysis," Microwave Opt. Tech. Lett., vol. 50, no. 10, pp. 2658-2662, Oct 2008.
6. Z.-G. Qian, and W.C. Chew, "Fast full-wave surface integral equation solver for multiscale structure modeling," IEEE Trans. Antennas Propag., vol. 57, no. 11, pp. 3594-3601, Nov 2009.

7. A. Bendali, "Numerical analysis of the exterior boundary value problem for the time-harmonic Maxwell equations by a boundary finite element method. II. The discrete problem," Math. Comp., vol. 43, no. 167, pp. 47-68, 1984

8. M. Benzi, G. H. Golub, and J. Liesen, "Numerical solution of saddle point problems," Acta Numerica, vol. 14, pp. 1-137, Apr. 2005.

9. L.J. Jiang and W.C. Chew, "A mixed-form fast multipole algorithm," IEEE Trans. Antennas Propag. AP-53 (12), pp. 4145-4156, 2005.

10. Y.G. Liu, W.C. Chew, L.J. Jiang, and Z.G. Qian, "A memory saving fast A-EFIE solver for modeling lowfrequency large-scale problems," submitted to APNUM.

11. Y.P. Chen, L.J. Jiang, Z.G. Qian, and W.C. Chew, "An augmented electric field integral equation for layered medium Green's function," in preparation.

12. E. Perrey-Debain, J. Trevelyan, and P. Bettess, "Wave boundary elements: A theoretical overview presenting applications in scattering of short waves," Eng. Analysis with Boundary Elements, vol. 28, pp. 131-141, Feb. 2004.

13. S. Langdon and S. N. Chandler-Wilde, "A wavenumber independent boundary element method for an acoustic scattering problem," SIAM J. Numer. Anal., vol. 43, no. 6, pp. 2450-2477, 2006

14. O. Bruno, C. Geuzaine, J. Monro, and F. Reitich, "Prescribed error tolerances within fixed computational times for scattering problems of arbitrarily high frequency: The convex case," Phil. Trans. R. Soc. London Series A, vol. 362, pp. 629-645, Mar. 2004.

15. W. C. Chew, J. M. Jin, E. Michielssen, and J. M. Song, (editors), Fast and Efficient Algorithms in Computational Electromagnetics, Artech House, Boston, MA, 2001.

16. R.F Harrington, Field Computation by Moment Methods, MacMillan, New York, 1968.

17. S.M. Rao, D.R. Wilton, and A.W. Glisson, "Electromagnetic scattering by surfaces of arbitrary shape," IEEE Trans Antennas Propagat AP-30, 409-418, 1982.

18. O. Ergul and L. Gurel, "A hierarchical partitioning strategy for an efficient parallelization of the multi-level fast multipole algorithm," IEEE Trans. Antennas Propag., vol. 57, no. 6, pp. 1740-1750, Jun 2009.

19. J.M. Taboada, L. Landesa, F. Obelleiro, J.L. Rodriguez, J.M. Bertolo, M.G. Araujo, J.C. Mourino, and A. Gomez, "High scalability FMM-FFT electromagnetic solver for super computer systems, " IEEE Antennas Propag. Mag., vol. 51, no. 6, pp. 20-28, Dec 2009.

20. C. P. Davis and W. C. Chew, "Frequency-independent scattering from a flat strip with TEz polarized fields," IEEE Trans. Ant. Propag., vol. 56, no. 4, pp. 1008-1016, April 2008.

21. W. E. I. Sha and W. C. Chew, "High frequency scattering by an impenetrable sphere," Progress In Electromagnetics Research, PIER 97, 291-325, 2009.

\footnotetext{
${ }^{1}$ The U of Hong Kong (e-mail: wcchew@hku.hk)

${ }^{2} \mathrm{U}$ of Illinois at Urbana-Champaign

${ }^{3}$ Synopsis Inc.

${ }^{4} \mathrm{U}$ of Rochester

${ }^{5}$ Schlumberger-Doll Research

${ }^{6}$ Intel Corp

${ }^{7}$ Raytheon UTD
} 